\title{
Can we develop wait lists for public health issues?
}

\author{
Nancy C. Edwards, Barbara L. Riley
}

W

ait lists have gained sharp prominence within the landscape of health care issues. Stories and statistics of how the common citizen has suffered and sometimes died while waiting for surgical and other medical procedures are legion, and they describe a failure of the health care system in a way that is easy to understand and that demands prompt and definitive attention.

However, there are also wait lists for public health services, and they rarely receive attention. These wait lists involve populations of citizens who have to wait (and sometimes die waiting) for clean water, adequate housing, safe transportation, and preventive and primary health care. For example, the wait times for sewage treatment facilities and potable water in some Northern Aboriginal communities run to ro or more years, and there is no doubt that many people have suffered during that period.

Public health wait lists are real but hidden or ignored. Increasing their visibility is essential for making priorities for public health services more compelling for politicians and the public. In turn, wait lists in public health would allow us to assess and address the longstanding imbalance in resources for acute care and public health services. ${ }^{1}$

Wait lists for medical procedures have become the measure of choice to assess how the Canadian health care system is doing, whether health services are operating effectively and efficiently, and whether taxpayers are getting value for money. Yet, in a pivotal report, wait lists in Canada were described as "non-standardized, capriciously organized, and poorly monitored." ${ }^{2}$ There were calls for major investments to improve management systems so that accurate and useful information can be delivered in a timely manner. Efforts to systematically document wait times across the acute care sector have grown. ${ }^{1,3-5}$ Wait lists for public health services ought to be included in these measurement systems and in public reports.

There are 2 fundamental questions regarding wait lists for public health services: What are they? And, if we do not make public health wait lists visible, what are the risks? Unlike wait lists in acute care, which predominantly describe the patient $\rightarrow$ numerators (patients who are seeking care but are unable to obtain timely services), public health wait lists are about the denominators (populations that need services, whether or not individuals within the population are seeking those services). Public health wait lists also reflect the wide range of health determinants targeted and services delivered by the health protection and preventive health sector. Thus, many public

health wait lists reflect the complex determinants of health experienced most forcefully by vulnerable populations. A noninclusive set of wait lists is shown in Box I. We invite readers to add their own examples to this list.

In the absence of discussion of public health wait lists and the presence of much discussion of acute care wait lists, governments put available resources into acute care rather than investing in public health services. The risk, and we would argue it is considerable, of not having public health wait lists to bring to the resource allocation table is that public health will not be at the table.

\section{Can wait lists for public health care services be measured?}

Hadorn and colleagues define wait lists as "a queue of patients who are deemed to need a health service that is in short supply relative to demand." We can readily apply elements of this definition to public health. Public health is a health service; public health professionals work with communities and patients whom they refer to as clients; and the supply of public health services is often woefully insufficient to yield substantial population health gains. ${ }^{1}$ Thus, a definition of public health wait lists would be "individuals and groups in the community who are in need of illness, injury and disease pre-

Box 1: Examples of public health wait lists and their corresponding population or health determinant

- Wait lists for policies to regulate reductions in carcinogenic exposures (exposure to environmental tobacco smoke in the workplace)

- Wait lists for physical activity programs among youth (students in senior grades of high school where there is no mandatory physical activity and a lack of affordable organized sports)

- Wait lists for subsidized housing (lack of low-cost housing)

- Wait lists for potable water (Aboriginal communities on reserves with contaminated drinking water supplies)

- Wait lists for safe and affordable day care (single-parent families without day care)

- Wait lists for essential and safe play areas (the working poor with no access to parks, safe playgrounds or affordable recreation services for their children) 
vention or health protection services that are in short supply relative to need." In Table I we suggest 4 dimensions for public health wait lists, using prevention and protection as 2 domains of public health services. Again, we invite readers to provide their input on these ideas.

Although wait lists may not be the most accurate platform for presenting the problems pervasive among the populations that require public health services, they are a means to make unmet and inequitable population health needs visible and prominent. The public health wait lists of today are the acute care wait lists of tomorrow. Ignoring wait lists for public health services fails the vulnerable population in the short term and the whole population in the long term. It would be naive to believe that public health wait lists alone will create the conditions sufficient for a more balanced approach to health care resource allocation. Nevertheless, developing a wait-list metric for use across both acute care and public health sectors would allow for better-informed decisions about that allocation. Perhaps most importantly, wait lists in public health would remind all of us that a primary goal of our health care system is to prevent illness and injury, and not just to treat the queue of patients requiring medical care.

Nancy Edwards is from the School of Nursing and Community Health Research Unit, University of Ottawa, Ottawa, Ont. Barbara Riley is from the Department of Health Studies and Gerontology, University of Waterloo, Kitchener, Ont.

Competing interests: None declared.

Contributors: Both authors contributed substantially to the conceptualization of the ideas in this article, wrote and revised the article critically for important intellectual content and approved the final version.

Acknowledgements: Manuscript preparation was supported by a personnel award to Barbara Riley from the Heart and Stroke Foundation of Canada and the Institute of Circulatory and Respiratory Health, Canadian Institutes of Health Research, and by a Nursing Chair to Nancy Edwards funded by the Canadian Health Services Research Foundation, Canadian Institutes of Health Research, and the Ontario Ministry of Health and Long-term Care.

Table 1: Proposed dimensions for wait lists in the public health sector with examples from 2 domains of public health services

Public health service domain; example

Dimension and definition

Burden of illness: The unmet burden of illness in the population. Typically described using epidemiologic indicators. Both immediate and longer-term projections of illness and disease burden need to be assessed.

\section{Inequitable burden of illness: The} unequal and unfair distribution of existing illness and disease, or illness and disease potential across population subgroups. Subgroups are disaggregated by characteristics such as sex, income, class and socioeconomic status.

Expected benefits (immediate and long term): Anticipated benefits resulting from the provision of effective intervention strategies.

Inequitable distribution of benefits: Anticipated unequal and unfair differential benefits by class, income, sex or other subgroup characteristic when effective interventions are applied.
- Incidence of influenza, measles and hepatitis A among nonimmunized populations.

- Prevalence of post-infectious disease complications among those not immunized (e.g., male sterility resulting from mumps, pneumonia following flu, pregnancy complications following rubella).

- Sex and income gradients in immunization coverage.

- Differential rates of post-flu pneumonia among low-income older adults living in crowded housing conditions and highincome older adults living in good housing conditions.

- Reduced incidence of disease and sequelae, both short and long term.

- Differential benefits due to uneven efficacy of vaccine distribution system with higher potential for breakdown of cold chain in remote areas with personnel not adequately trained in cold chain maintenance.

- Differential uptake of flu immunization among caregivers due to presence or absence of employment requirements and support for immunization.
- Environmental tobacco smoke policies in the workplace reduce second-hand smoke exposure and increase smoking cessation rates.

- Environmental tobacco smoke policies in public places reduce second-hand smoke exposure. Benefits are influenced by strength and coverage of restrictions and by patterns of enforcement.

- Differential benefits of environmental tobacco smoke policies in workplaces when certain workplaces are exempt from regulations (e.g., bars, bingo halls).

- Differential benefits from environmental tobacco smoke policy due to uneven policy enforcement. 


\section{REFERENCES}

I. Bennett J. Investment in population health in five OECD countries. OECD Health Working Paper \#2. Paris: Organization for Economic Cooperation and Development; 2003.

2. McDonald $\mathrm{P}$, Shortt $\mathrm{S}$, Sanmartin $\mathrm{C}$, et al. Waiting lists and waiting times for health care in Canada: more management!! More Money?? Ottawa: Health Canada; I998.

3. Tu JV, Shortt S, McColgan P, et al. Reflections and recommendations. In: Tu JV, Pinfold SP, McColgan P, Laupacis A, editors. Access to health services in Ontario. ICES Atlas. Toronto: Institute for Clinical Evaluation Sciences; 2005.

4. Western Canada Waiting List Project. Moving forward (Final report). Calgary: The
Project; 2004. p. I-47.

5. Van Doorslaer E, Masseria C. Income-related inequality in the use of medical care in 21 OECD countries. OECD Health Working Paper \# I4 $_{4}$ Paris: Organization for Economic Cooperation and Development; 2004.

6. Hadorn DC and the Steering Committee of the Western Canada Waiting List Project. Setting priorities for waiting lists: defining our terms. $C M A J$ 2000;163(7):857-6o.

Correspondence to: Dr. Nancy C. Edwards, University of Ottawa, School of Nursing, 45I - III8 Smyth Rd., Ottawa ON KIH 8M5; fax 613 562-5658; nancy.edwards@uottawa.ca

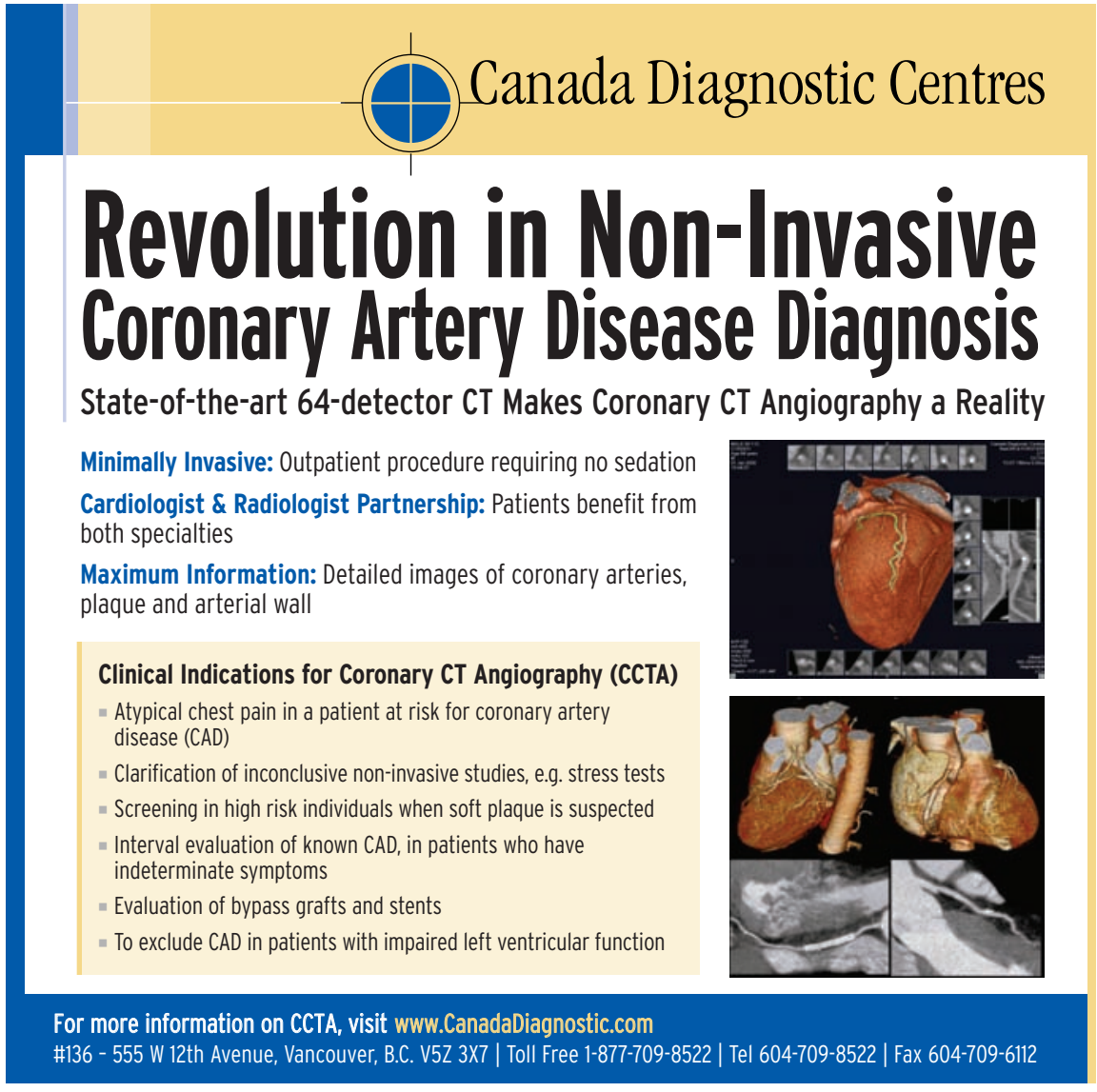

